\title{
Molecular Simulation Studies of Hydrogen Enriched Methane (HEM) Storage in Covalent Organic Frameworks
}

Jiawei Liao, ${ }^{a}$ A. Ozgur Yazaydin, ${ }^{b}$ Siyuan Yang, ${ }^{a}$ Fan Li, ${ }^{c}$ Lifeng Ding ${ }^{* a}$

${ }^{a}$ Department of Chemistry, Xi'an JiaoTong-Liverpool University, 111 Ren'ai Road, Suzhou Dushu Lake Higher Education Town, Jiangsu Province, 215123, China

${ }^{\mathrm{b}}$ Department of Chemical Engineering, University College London, Torrington Place, London, WC1E 7JE, United Kingdom

${ }^{\mathrm{c}}$ Electricity Transmission Asset Management, National Grid House, Warwick, CV34 6DA, United Kingdom

*To whom correspondence should be addressed. E-mail: Lifeng.Ding@xjtlu.edu.cn 


\section{Abstract}

Instead of separating hydrogen and methane mixture, such as synthetic gas, to obtain pure hydrogen and methane as clean fuel, hydrogen enriched methane (abbreviated as HEM) storage in 33 different Covalent Organic Frameworks (COFs) were studied for the first time near ambient temperatures using Grand Canonical Monte Carlo (GCMC) simulation. The use of HEM for on-board combustion engine is also known to be able to improve combustion performance as well as decrease noxious emissions. HEM adsorption performance in the COFs was mainly evaluated from three different aspects: volumetric energy density of combustion of stored HEM, gravimetric energy density of combustion of stored HEM and hydrogen selectivity. Several properties of the COFs, such as surface area, porosity, pore size were calculated for establishing the correlation with the HEM adsorption performance. The effect of temperature, initial hydrogen/methane bulk composition and hydrogen and methane/hydrogen's heat of adsorption (HOA) in COFs on the performance of HEM adsorption were also investigated. Our work suggested there exists a complex interplay of the properties of the COFs, temperature and bulk composition which influence the energy density of the adsorbed HEM as well as methane and hydrogen ratio in the adsorbed phase.

Keywords: hydrogen enriched methane storage; Covalent Organic Framework; hythane; molecular simulations 


\section{Introduction}

With emphasis on promoting clean energy use in recent years, the research of finding alternative environmental friendly fuels has attracted wide interest. Methane, as an alternative clean fuel that has little sulfur/nitrogen pollutant emission, as well as lower carbon emission compared to petroleum-based fuels, is slowly but gradually expanding its on-board applications.[1] Up to now cost intensive liquefied or compressed methane has found application in public transportation,[2] but wide application of methane storage as an alternative on-board fuel has not been achieved yet . Sun et. al, suggested that mixing hydrogen into natural gas could increase the combustion performance by $15 \%$ in a natural gas powered engine.[3] Karim and coworkers suggested that the flame initiation speed and flame propagation rate of hydrogen/methane mixtures could be improved significantly in the engine.[4] The use of hydrogen enriched compressed natural gas (HCNG) was suggested to greatly reduce noxious emissions, such as $\mathrm{NO}_{\mathrm{x}}$ and $\mathrm{CO}$, in the engine.[5] Hydrogen and methane mixture with a composition of $20 \%$ hydrogen and $80 \%$ methane by volume as a new fuel was coined as a new brand name, Hythane ${ }^{\circledR}$.[6] HEM studied in this paper allows to consist of any arbitrary proportion of hydrogen and methane. Extensive efforts have been devoted into the separation of hydrogen and methane from synthetic gas in order to obtain purified hydrogen or methane.[7] The direct use of methane/hydrogen mixture as fuel, such as the product of synthetic gas will save the cost of separation of methane and hydrogen. The conventional approaches to store hydrogen and methane for on-board use, such as liquefied or compressed gas storage, 
require extreme conditions which are cost intensive as well as are associated with safety concerns.[2] Adsorption based hydrogen and methane storage have emerged as an alternative approach for on-board energy gas storage.

There have been several studies focusing on using carbonaceous porous materials to store hydrogen enriched methane.[6],[8],[9] P. Kowalczyk's et. al,[6] and Kumar et. al,[9] indicated that pore width and pore geometry of carbonaceous porous materials have significant effects on the selectivity of adsorbed methane over hydrogen. Confined space in small pores of carbon material will strongly adsorb methane and deplete hydrogen. Larger pore space will ease this effect and promote more hydrogen adsorption. Morales-Cas et. al, suggested that the existence of methane molecules could be helpful to the stabilization of hydrogen adsorption in second and higher adsorption layers.[8] Many porous carbonaceous materials, such as active carbon and carbon nanofoam, have disordered structures. It is challenging to have ordered pore size and geometry. Even for carbon nanotubes it is difficult to maintain a regular inter-nanotube space. Being able to fine tune the pore structure can help maintain a stable adsorbed methane/hydrogen ratio as well as promote HEM adsorption capacity. The newly emerged perfectly ordered crystalline nanoporous materials, such as Covalent Organic Frameworks (COFs),[10] Metal Organic Frameworks (MOFs),[11] and Zeolitic Imidazolate Frameworks(ZIFs),[12] could be promising for HEM storage. Among them, COFs are a novel class of crystalline porous polymers that are mainly composed of light elements $(\mathrm{B}, \mathrm{C}, \mathrm{N}, \mathrm{O})$ connected by strong covalent bonds. Compared to MOFs and ZIFs, COFs have the advantages of 
lower densities and better chemical and thermal stability.[13]'[14] Recent studies showed that COFs are among the highest performing materials for single component adsorption of gases, such as methane[15] and hydrogen[16].

In this study, molecular simulations were used to study HEM storage performance of 33 different COFs. HEM adsorption performances of these COFs are mainly evaluated from following aspects. Firstly, instead of evaluating total methane/hydrogen mixture adsorption capacity, we use total heat of combustion provided by the adsorbed methane/hydrogen in COFs to measure energy aspect of HEM adsorption performance in COFs. Secondly, it is true that hydrogen adsorption capacity is usually much lower than methane adsorption capacity in nanoporous materials,[6] particularly at near room temperature. Moderate level of hydrogen storage ratio is needed in HEM in order to bring substantial improvement of the combustion efficiency in the engine. To identify COFs with good hydrogen adsorption selectivity is our second priority. Moreover, we aim to investigate the correlation between the COFs' structural characteristics and HEM's adsorption performance. Such established correlation would allow the design of novel COFs' with desirable characteristics and performance.

\section{Computational Details}

\subsection{COFs structures}

In this work, 33 different COFs were considered which included more than $4 \mathrm{COF}$ subfamilies (e.g. CTFs (CTF stands for covalent triazine-based framework), Tp-COFs (Tp stands for either triphenylene or triformylphloroglucinol), NPNs (NPN stands for 
nitroso polymer networks), Star-COFs (star shaped COFs)) and other COFs, such as, COF-102, COF-103, COF-105 and COF-108,[17] featuring high methane uptake at ambient conditions. Model atomistic structures were constructed according to the experimental crystallographic data. Geometric surface area, porosity and the pore size of the COFs considered in this work vary diversely. Overall, the volumetric geometric surface area of these COFs ranges from $675 \mathrm{~m}^{2} / \mathrm{cm}^{3}$ to $2253 \mathrm{~m}^{2} / \mathrm{cm}^{3}$; the porosity ranges from $34.75 \%$ to $93.69 \%$ and the dominant pore size ranges from $3.465 \AA$ to 43.895A. Topologies of the 33 COFs included 2D COFs with hexagonal and tetragonal topologies, such as Star-COF and Zn-pcpy-COF, and 3D-COFs with $(3,4)$-connected ctn and bor nets and diamond net (dia) topologies, such as, COF-102, COF-103 and COF-108. Full data for the structural properties of the COFs are provided in Table S1.

\subsection{GCMC simulations}

In this study, Grand Canonical Monte Carlo (GCMC) simulations were employed to study methane and hydrogen co-adsorption behavior in COFs. All GCMC simulations were carried out using RASPA package.[18] Lennard-Jones (LJ) potential (equation1) was used to treat the van der Waals interactions between adsorbates and adsorbents. LJ parameters between different types of atoms were calculated using Lorentz-Berthelot mixing rules (equations 2 and 3).

$$
\begin{aligned}
& U_{i j}=4 \varepsilon_{\mathrm{ij}}\left[\left(\frac{\sigma_{\mathrm{ij}}}{r_{i j}}\right)^{12}-\left(\frac{\sigma_{\mathrm{ij}}}{r_{i j}}\right)^{6}\right] \\
& \sigma_{\mathrm{ij}}=\frac{1}{2}\left(\sigma_{\mathrm{i}}+\sigma_{\mathrm{j}}\right) \\
& \varepsilon_{\mathrm{ij}}=\sqrt{\varepsilon_{\mathrm{i}} \varepsilon_{\mathrm{j}}}
\end{aligned}
$$


where, $i$ and $j$ are interacting atoms, and $r, \varepsilon$ and $\sigma$, are atom-atom distance, LJ well depth and the representative distance for the interactions, respectively. In this work, LJ potential parameter values of COF framework atoms were taken from the DREIDING force field.[19] All force field parameters for both COFs and guest gas molecules are given in Table S3 and S4. Methane was modeled as a single site molecule and the LJ potential parameters of $\mathrm{CH}_{4}\left(\sigma_{\mathrm{CH}_{4}}=3.73 \AA\right.$ and $\left.\varepsilon_{\mathrm{CH}_{4}} / \mathrm{k}_{\mathrm{B}}=148.0 \mathrm{~K}\right)$ were taken from TraPPE force field.[20] Hydrogen was modeled as a single site molecule as well. The LJ parameters of hydrogen $\left(\sigma_{\mathrm{H}_{2}}=2.96 \AA\right.$ and $\left.\varepsilon_{\mathrm{H}_{2}} / \mathrm{k}_{\mathrm{B}}=34.2 \mathrm{~K}\right)$ were taken from the work of Buch.[21] According to Garberoglio's et. al,[22] the influence of electrostatic interactions for $\mathrm{H}_{2}$ and $\mathrm{CH}_{4}$ adsorption in MOFs is negligible. Point charge interactions in this work are ignored. The cutoff radius for LJ interaction was set to be $12.8 \AA$. Due to the importance of quantum diffraction effects for $\mathrm{H}_{2}$ adsorption at cryogenic temperatures, $\mathrm{H}_{2}$ adsorption at $77 \mathrm{~K}$ was simulated with the quasiclassical Feynman-Hibbs (FH) corrected potential.[23]

All GCMC simulations included a $1 \times 10^{5}$ cycle equilibration run followed by a $1 \times 10^{5}$ cycle production run. Each cycle included $\mathrm{N}$ steps where $\mathrm{N}$ was the number of adsorbed molecules in the system, which fluctuates during the GCMC simulations. For each step, random translation moves, insertion/deletion moves were sampled to calculate the ensemble averages. All COF framework atoms were kept rigid during the simulation. Geometric surface area of COFs was calculated according to Duren's method.[24] For the pore volume determination of COFs, we adopted the calculation method published by Talu and Myers.[25] The isosteric heat of adsorption (HOA) of 
pure methane and pure hydrogen adsorbed in COFs were calculated at low loadings following the fluctuations of energy/particle in the grand canonical ensemble in this study.[26] Finally, the pore-size distribution of COFs was obtained using the method proposed by Gubbins et al.[27]

\subsection{Energy storage and selectivity}

The adsorbed amount of hydrogen and methane by GCMC simulation is absolute uptake $\mathrm{N}_{\mathrm{ab}}$, while the experimentally measured adsorption amount is excess adsorption $\mathrm{N}_{\mathrm{ex}}$. The relationship between them is $\mathrm{N}_{\mathrm{ex}}=\mathrm{N}_{\mathrm{ab}}-\rho_{\mathrm{b}} \mathrm{V}_{\text {pore }}$, where $\rho_{\mathrm{b}}$ is the gas density in pure gas phase, $\mathrm{V}_{\text {pore }}$ is the pore volume. The total energy of HEM stored in COFs is evaluated via calculating the total volumetric or gravimetric energy of the total predicted methane and hydrogen stored in COFs by GCMC simulations. Here, we define volumetric and gravimetric energy density of HEM adsorption in COFs by:

$\mathrm{E}_{\mathrm{v}}=\mathrm{NCH}_{4}(\mathrm{v}) \times\left(-\Delta \mathrm{HCH}_{4}\right)+\mathrm{NH}_{2}(\mathrm{v}) \times\left(-\Delta \mathrm{HH}_{2}\right)$

$\mathrm{E}_{\mathrm{g}}=\mathrm{N}_{\mathrm{CH}_{4}(\mathrm{~g})} \times\left(-\Delta \mathrm{HCH}_{4}\right)+\mathrm{N}_{2}(\mathrm{~g}) \times\left(-\Delta \mathrm{HH}_{2}\right)$

where $\mathrm{N}_{\mathrm{CH}_{4}(\mathrm{v})}$ and $\mathrm{NH}_{2}$ (v) are the volumetric adsorption uptake of two components in moles per unit volume of the COF framework; $\mathrm{N}_{\mathrm{CH}_{4}(\mathrm{~g})}$ and $\left.\mathrm{N}_{\mathrm{H}_{2}} \mathrm{~g}\right)$ are the gravimetric adsorption uptake of two components, in moles per unit mass of the COF framework; $\Delta \mathrm{HCH}_{4}$ and $\Delta \mathrm{HH}_{2}$ denotes the heat of methane and hydrogen combustion, respectively. According to Dell et. al, heat of combustion of methane and hydrogen's are -890.3 $\mathrm{kJ} / \mathrm{mol}$ and $-285.8 \mathrm{~kJ} / \mathrm{mol}$, respectively.[28] On the other hand, it is crucial as well as challenging to maintain the ratio of adsorbed hydrogen in HEM storage system.[8] In 
Kowalczyk et. al's work, $90 \%$ of bulk mole fraction of hydrogen in hydrogen/methane mixture is needed, in order to obtain merely $7 \%$ of adsorbed hydrogen by energy in slitlke carbon.[6] Evaluating hydrogen adsorption selectivity in COFs is also one important factor to judge COF's HEM adsorption performance. Hydrogen adsorption selectivity in this work is defined by:

$\mathrm{S}=\left(\mathrm{XH}_{2} / \mathrm{XCH}_{4}\right)\left(\mathrm{YCH}_{4} / \mathrm{YH}_{2}\right)$,

where $\mathrm{x}$ and $\mathrm{y}$ are the mole fractions of methane and hydrogen in the adsorbed and bulk phase respectively.

\section{Result \& Discussion}

\subsection{Validation of the force field}

To validate the employed force field used in this work, simulated excess adsorption isotherms of methane and hydrogen in COF-6, COF-10 and COF-102 were compared with the experimental data as shown in Fig.1.[22],[17] Simulated isotherms of methane and hydrogen adsorption show good agreement with the experimental isotherms. Only the simulated methane excess uptake in COF-102 is slightly higher than the experimental result. This could be due to incomplete solvent removal in the COF or possible defects.

\subsection{Screening of HEM adsorption in 33 COFs}

HEM adsorption isotherms in $33 \mathrm{COFs}$ were simulated at $298 \mathrm{~K}$ with the pressure ranging from 0.01 bar to 85 bar. The composition of the bulk hydrogen and methane mixture was set to be equimolar, e.g. a ratio of 1:1. Fig.2 shows that methane uptake in COFs is much higher than hydrogen uptake at $298 \mathrm{~K}$ for across the whole pressure 
range studied. (Fig.2 (d) provides methane and hydrogen adsorption isotherms in COF-102, COF-105, COF-108 and NPN-1.) The hydrogen selectivities in all the COFs are smaller than 1 as given in Fig.2 (c), which indicates that the adsorbed hydrogen ratio is always smaller than that of in the bulk mixture. Among them, COF-105 and COF-108 are two COFs with the highest hydrogen selectivity (over 0.4). The energy densities of HEM stored in COFs are mainly dominated by methane loading in COFs due to the relatively lower hydrogen uptake as well as low molar heat of combustion of hydrogen. As shown in Fig.2 (d), the trend of total energy density (ED) fit well with the trend of methane uptake. Both gravimetric and volumetric energy density isotherm in NPN serial COFs quickly reaches plateau around 10 bar indicating methane adsorption in NPN series COFs can be quickly saturated at a pressure close to atmospheric pressure at room temperature. It is worth noting that although the gravimetric energy densities in NPN series COFs are relatively lower than those of in other COFs, their volumetric energy density easily outperform other COFs for pressures below 40 bar. Energy density isotherms in other COFs, such as, COF-102, COF-105 and COF-108 increase steadily with the pressure up to 85 bars. As shown in Fig.2. (a) and (b), the total stored gravimetric energy density of HEM in COF-102, COF-103, COF-105 and COF-108, can exceed 2015 target established by Department of Energy (DOE) in US, $(6480 \mathrm{~kJ} / \mathrm{kg})$ above $30 \mathrm{bar}$ pressure and the total stored volumetric energy density of HEM in COF-102, COF-103 and NPN-1, can exceed 2015 DOE target (4680 kJ/L)above 40 bar pressure.[29] 
As mentioned above, total energy density of HEM adsorption and hydrogen adsorption selectivity are two main factors to evaluate the performance of HEM adsorption in COFs. Hydrogen selectivity was plotted against both gravimetric and volumetric energy densities for all 33 COFs at three different pressures: 1bar, 35bar and $80 \mathrm{bar}$ at $298 \mathrm{~K}$ in Fig.3. The ideal COFs should be located in the upper right corner of the figures, which will have both high hydrogen selectivity and large energy density.

By analyzing these diagrams, it can be found that it is difficult to find COFs that have both good hydrogen selectivity and good energy density at 1 bar pressure. NPN type of COFs possess both high gravimetric and high volumetric energy density at 1 bar, while their hydrogen selectivities are among the lowest ones. COFs that have relatively high $\mathrm{H}_{2}$ selectivity, such as COF-105, COF-108, COF-43, Star-COF-1 and Star-COF-2, give relatively low gravimetric and volumetric energy density at 1 bar. When the pressure is increased to medium pressure (35 bar) and high pressure (80 bar), many COFs are moving towards to the upper-right corner of the diagram where both the energy density and hydrogen selectivity are high. COF-105 and COF-108 exceeds all other COFs in terms of gravimetric energy density and hydrogen selectivity at medium and high pressures. Nevertheless, in terms of volumetric energy density, which should play more significant role for on-board fuel applications, COF-105 and COF-108 lose their leading positions. COF-102 and COF-103 are among the leading COFs with highest volumetric energy density. However, the hydrogen selectivities of COF-102 and COF-103 are not very promising, only around 
0.15 at $298 \mathrm{~K}$. Overall, for all the $33 \mathrm{COFs}$, it is possible to find COFs, such as COF-105 and COF-108, with both large gravimetric energy density and high hydrogen selectivity.

\subsection{The Effect of Structural Characteristics of COFs}

To establish and understand the correlation between COFs' structural characteristics as well as the interaction strength of methane/hydrogen with COFs, and COFs' HEM adsorption performance is of particular significance for the future design of the ideal COFs to be used in HEM storage.

\subsubsection{The Effect of heat of adsorption}

The isosteric heats of adsorption (HOA) of methane and hydrogen in COFs are calculated and provided in Table S2. In this study, all the values of HOA were taken in positive absolute value although the adsorption is an exothermic process. Overall, the HOA of methane in COFs is around three to four times higher than the HOA of hydrogen in COFs. Such large HOA difference is the main reason that leads to the relatively low hydrogen selectivity. As shown in Fig.4 (a), hydrogen selectivity is roughly inversely proportional with the HOA differences of methane and hydrogen in COFs. On the other hand, from Fig.4 (b) we also observe that the volumetric energy density of HEM adsorption in COFs increases almost linearly with the HOAs of methane in COFs at 1 bar pressure with the exception of NPN type COFs, whose volumetric energy densities are much higher than the rest of COFs. At higher pressures such linear correlation is weakened between HOA and volumetric energy density. As mentioned above, the total energy density of COFs is mainly affected by 
methane uptake. At low pressures, the dominant factor responsible for methane uptake is the HOA, which is indirectly related to the volumetric energy density of HEM adsorption.

\subsubsection{The Effect of Surface Area}

With the increase of pressure, the volumetric energy density of HEM adsorption in COFs starts to correlate closely with COFs' volumetric geometric surface area. As shown in Fig.5, apart from NPN type COFs and COF-1, which have exceptionally high HOA with methane and hydrogen, the slope of the linearly increasing tendency of volumetric energy density and volumetric surface area can be enhanced by the rising pressure. At higher pressure, such as 80 bar, the energy density increases faster with the increase of COFs' surface areas. This can be explained with the fact that at low pressure the surface area of COFs has not been fully covered by adsorbates. The adsorption mainly happens at strong interaction sites. With the increase of the pressure, adsorbates start to cover the surface area of COFs.

\subsubsection{The Effect of Porosity and Pore Size}

According to Bae et al.[30] the porosity of MOFs or COFs plays a significant role on both methane and hydrogen adsorption at the high pressure region. It is generally true that high pressure will trigger multi-layer adsorption to allow adsorbed methane and hydrogen molecules to explore the central void space. Both gravimetric energy density and volumetric energy density of HEM adsorption in different COFs are plotted against porosity at $298 \mathrm{~K}$ and 80 bar in Fig.6 (a) and (b). It is observed that the gravimetric energy density increases roughly proportional to the porosity of the COFs. 
Surprisingly, the volumetric energy density has almost no correlation with COFs' porosity. COF-105 and COF-108, whose volumetric energy densities are only half of those of COF-102 and COF-103, share similar porosity with COF-102 and COF-103. This is due to a synergic effect of both surface area and porosity on HEM adsorption at 80 bar pressure. As discussed above, surface area actually still plays a significant role on promoting HEM uptake at 80 bar. It can be seen from Fig.6 (a) that the gravimetric surface area increases roughly linearly with the porosity of the COFs. Both the gravimetric surface area and the porosity will enhance the gravimetric energy density of the COFs. Nevertheless, high porosity does not necessary mean high volumetric surface area. As can be seen from Fig.6 (b), the porosity has almost no correlation with the volumetric surface area of the COFs. COFs with high porosities (up to 92.8\%), such as COF-105 and COF-108, could have low volumetric surface areas (around $1100 \mathrm{~m}^{2} / \mathrm{cm}^{3}$ ). Such interplay of the porosity and the volumetric surface area leads to a poor correlation of porosity and volumetric energy density of adsorbed HEM.

On the other hand, both the porosity and the dominant pore size of the COFs are important factors that affect hydrogen selectivity. Fig.6 (c) and (d) give scatter diagrams of the $\mathrm{H}_{2}$ selectivity against both the porosity and dominant pore size of 33 COFs at $298 \mathrm{~K}$ and 80 bar. It is observed that hydrogen selectivity is in a linear correlation with both porosity and the dominant pore size of the COFs. Such finding is in good agreement with Kumar et al[9] and Morales-Cas et al's[8] work. Narrow pores in COFs will strongly adsorb methane due to its larger interaction strength with 
COFs and deplete hydrogen. For instance, NPN type COFs which possess pores ranging from 3 to $5 \AA$ have extremely small hydrogen selectivity. Large pore space will ease this effect. It should be however noted that COF-103 and COF-102 are two outliers that deviate from the linear relation between the hydrogen selectivity and the porosity. COF-105, COF-108 and COF-43 are also the obvious outliers for the linear correlation of the hydrogen selectivity and the dominant pore size. It is again due to the interplay of the porosity and the dominant pore size of the COFs. Star-COF-1 and Star-COF-2 which have both high porosity and large dominant pore size show good hydrogen selectivity. COF-102 and COF-103 have high porosity, but they have relatively small dominant pore size (below $15 \AA$ ) so they appear below the trend line. COF-105, COF-108 and COF-43 that possess dominant pores above $15 \AA$ as well as leading porosities enable them to have top hydrogen selectivities. As can be seen from the HEM adsorption snapshots in COF-108 at 298K (Fig.S1), adsorbed $\mathrm{H}_{2}$ molecules tend to appear more often in the center of the pore void then the surface of the COF.

\subsection{The Effect of Temperature}

\subsubsection{To Volumetric energy density}

To further explore the possibilities of improving volumetric energy density of HEM storage as well as hydrogen selectivities of COFs, we allow both the temperature to vary from $250 \mathrm{~K}$ to $350 \mathrm{~K}$ and methane/hydrogen bulk ratio to vary from 10:90 to 90:10. Three distinctively different COFs are selected for this part of the study: NPN-1 with good volumetric energy density, but extremely small hydrogen selectivity at 298K; COF-103 with good volumetric energy and intermediate hydrogen 
selectivity at 298K; and COF-108 with top hydrogen selectivity and relatively low volumetric energy density at $298 \mathrm{~K}$. The volumetric energy density and hydrogen selectivities of HEM adsorption isobars of these 3 COFs at 3 different pressures ( 1 bar, 35 bar, 80 bar) are established in Fig.7, In general, it is observed that decreasing temperature is helpful to raise the volumetric energy density in Fig.7 (a), (b) and (c). Meanwhile, the hydrogen selectivity is reduced (Fig.7 (d)). At 1 bar, the change of temperature or hydrogen/methane bulk ratio affects the volumetric energy density of NPN-1 only due to the strong heat of adsorption of methane in NPN-1. Considering the low interaction strength of methane and hydrogen with COF-103 and COF-108, changing the temperature or hydrogen/methane bulk composition at 1 bar pressure barely affects their hydrogen or methane uptake. At higher pressures of 35 bar and 80 bar, the change of temperature dramatically affects the volumetric energy density of COF-103 and COF-108. At 35 bar, changing the temperature from $298 \mathrm{~K}$ to $250 \mathrm{~K}$ could almost double the volumetric energy density of COF-103, but the hydrogen selectivity is compromised to half of that at $298 \mathrm{~K}$. The drop in the temperature enhances gas adsorption in porous materials.

Considering that hydrogen selectivity is fairly low in all the COFs studies, one approach to raise the adsorbed hydrogen content is to raise the hydrogen bulk composition in the bulk phase. However, due to the low energy density of hydrogen, increasing the adsorbed hydrogen content inevitably decreases the total volumetric energy density. As shown in Fig.8 (b) and (c), the volumetric energy densities of COF-103, COF-108 decrease by about two thirds at 35 bar and 80 bar pressure and 
298K temperature when the mol fraction of hydrogen in the bulk phase increased from $10 \%$ to $90 \%$. The volumetric energy density of NPN-1 has a large volumetric energy density drop at 1 bar pressure when hydrogen bulk mol fraction is increased. In order to allow hydrogen contribution in HEM fuel to exceed $7 \%$ by energy, which is required to form an effective HEM fuel,[6] more than $40 \%$ and $60 \%$ of hydrogen mol fraction are needed for COF-108 and COF-103, respectively, at $298 \mathrm{~K}$ and 35 bar.

\section{Conclusion}

In summary, this work has brought new insight into the potential HEM fuel storage in COFs. For all 33 COFs studied, it is found that HEM adsorption in many COFs, such as, COF-102, COF-103, COF-105, COF-108, could easily reach high gravimetric energy density, which exceeds 2015 DOE target, when the pressure reach 35 at $298 \mathrm{~K}$ due to the light weight of COFs. There are also COFs, such as, COF-105 and COF-108, whose HEM adsorption with methane/hydrogen bulk phase ratio 1:1 will also exceed volumetric energy density of 2015 DOE target with pressures over 40 bar at $298 \mathrm{~K}$. One challenging issue to store HEM in COFs is to raise the hydrogen selectivity. Overall, hydrogen selectivity in COFs is relatively low at room temperature. COF-105 and COF-108 are two COFs with the highest hydrogen selectivity due to their large porosity and pore sizes. Increasing the hydrogen fraction in the bulk phase enhances the hydrogen fraction in the adsorbed phase. Nevertheless, due to the fact that energy density is dominated by the adsorbed methane, increasing adsorbed hydrogen inevitably decreases the energy density of adsorbed HEM in COFs. Both the energy density of adsorbed HEM and hydrogen selectivity in COFs are 
affected by an interplay of the HOA of methane/hydrogen in COFs, surface area, porosity and pore size of the COFs. Overall, high HOA of methane in COFs and surface area promote energy density of adsorbed HEM in COFs. It is helpful to increase the hydrogen selectivity in COFs by matching hydrogen's HOA to methane's HOA in COFs. COFs with large pores and high porosity also enhances the hydrogen selectivity in COFs.

\section{ASSOCIATED CONTENT}

Supplementary Information (SI) available: COF models, force field parameters, absolute hydrogen and methane uptake isotherms in the COFs. This material is available free of charge via the Internet at http://pubs.acs.org.

\section{AUTHOR INFORMATION}

\section{Corresponding Author}

E-mail: Lifeng.Ding@xjtlu.edu.cn

\section{ACKNOWLEDGEMENT}

The authors acknowledge financial support from Chinese Young Scholar National

Science Foundation Grant (21403171) and Xi'an JiaoTong-Liverpool University

Research Development Fund (PGRS-13-03-08), computational resources from Shenzhen Cloud Computing Center.

\section{References}


[1] Y. He, W. Zhou, G. Qian, B. Chen, Chem Soc Rev 43 (2014) 5657-5678.

[2] W. Zhou, Chem. Rec. 10 (2010) 200-204.

[3] Y. Sun, Y.P. Zhou, L. Zhou, X.P. Gao, H.T. Yuan, Chem. Phys. Lett. 357 (2002) 287-292.

[4] G. a. Karim, I. Wierzba, Y. Al-Alousi, Int. J. Hydrogen Energy 21 (1996) 625-631.

[5] K. Nanthagopal, R. Subbarao, T. Elango, P. Baskar, K. Annamalai, Therm. Sci. 15 (2011) 1145-1154.

[6] P. Kowalczyk, S.K. Bhatia, J. Phys. Chem. B 110 (2006) 23770-23776.

[7] M.C. Mitchell, M. Gallo, T.M. Nenoff, J. Chem. Phys. 121 (2004) 1910-1916.

[8] A.M. Morales-Cas, C. Moya, B. Goto, L.F. Vega, G. Calleja, J. Phys. Chem. C 111 (2007) 6473-6480.

[9] K.V. Kumar, E. a. Müller, F. Rodríguez-Reinoso, J. Phys. Chem. C 116 (2012) 11820-11829.

[10] X. Feng, X. Ding, D. Jiang, Chem. Soc. Rev. 41 (2012) 6010.

[11] S. Ma, H.-C. Zhou, Chem. Commun. 46 (2010) 44-53.

[12] K.S. Park, Z. Ni, A.P. Côté, J.Y. Choi, R. Huang, F.J. Uribe-Romo, H.K. Chae, M. O’Keeffe, O.M. Yaghi, Proc. Natl. Acad. Sci. U. S. A. 103 (2006) 10186-10191.

[13] Y. Liu, D. Liu, Q. Yang, C. Zhong, J. Mi, Ind. Eng. Chem. Res. 49 (2010) 2902-2906.

[14] M. Tong, Q. Yang, C. Zhong, Microporous Mesoporous Mater. 210 (2015) 142-148.

[15] J.L. Mendoza-Cortes, T. a. Pascal, W. a. Goddard, J. Phys. Chem. A 115 (2011) 13852-13857.

[16] S.-Y. Ding, W. Wang, Chem. Soc. Rev. (2013) 548-568.

[17] H. Furukawa, O.M. Yaghi, J. Am. Chem. Soc. 131 (2009) 8875-83.

[18] D. Dubbeldam, S. Calero, D.E. Ellis, R.Q. Snurr, Mol. Simul. 42 (2015) 81-101.

[19] S.L. Mayo, B.D. Olafson, W.A.I. Goddard, J. Phys. Chem. 101 (1990) 8897-8909.

[20] M.G. Martin, J.I. Siepmann, J. Phys. Chem. B 102 (1998) 2569-2577.

[21] V. Buch, J. Chem. Phys. 100 (1994) 7610.

[22] G. Garberoglio, A.I. Skoulidas, J.K. Johnson, J. Phys. Chem. B 109 (2005) 13094-13103.

[23] J. Liu, J.T. Culp, S. Natesakhawat, B.C. Bockrath, B. Zande, S.G. Sankar, G. Garberoglio, J.K. Johnson, J. Phys. Chem. C 111 (2007) 9305-9313.

[24] T. Düren, L. Sarkisov, O.M. Yaghi, R.Q. Snurr, Langmuir 20 (2004) 2683-2689.

[25] O. Talu, A.L. Myers, AIChE J. 47 (2001) 1160-1168.

[26] F. Karavias, a L. Myers, Langmuir 7 (1991) 3118-3126.

[27] L.D. Gelb, K.E. Gubbins, Langmuir 15 (1999) 305-308.

[28] R.M. Dell, D.A.J. Rand, Clean Energy, The Royal Society of Chemnistry, Cambridge, 
UK, 2004.

[29] US Department of Energy, Targets for on-board hydrogen storage systems: current R\&D focus is on 2015 targets with potential to meet ultimate targets. < http://energy.gov/sites/prod/files/2015/05/f22/fcto_targets_onboard_hydro_storage_explan ation.pdf >

[30] Y.-S. Bae, R.Q. Snurr, Microporous Mesoporous Mater. 135 (2010) 178-186.

Fig.1. Experimental and simulated excess $\mathrm{CH}_{4}$ and $\mathrm{H}_{2}$ adsorption: (a) $\mathrm{CH}_{4}$ in $\mathrm{COF}-6$ and COF-102 at 298K; (b) $\mathrm{H}_{2}$ in COF-6 and COF-10 at 77K[22],[17] (EXP = experimental results; $\mathrm{SIM}=$ simulation results)

Fig. 2. Simulated HEM adsorption of 9 representative COFs at 298K: (a) gravimetric energy density isotherms of adsorbed HEM; (b) volumetric energy density isotherms of adsorbed HEM; (c) hydrogen adsorption selectivity with pressure; (d) volumetric adsorption isotherms of hydrogen and methane compared with the volumetric energy density isotherms $\left(\mathrm{CH}_{4}=\right.$ volumetric methane adsorption isotherm, $\mathrm{H}_{2}=$ volumetric hydrogen adsorption isotherm, $\mathrm{ED}$ $=$ volumetric energy density isotherm)

Fig. 3. The scatter diagram of energy density against hydrogen adsorption selectivity of the 33 COFs at $298 \mathrm{~K}$ : gravimetric energy density vs. hydrogen selectivity: at (a) 1 bar, (b) 35 bar, (c) 80 bar; volumetric energy density vs. hydrogen selectivity: at (d) 1 bar, (e) 35 bar, (f) 80 bar.

Fig.4. (a) Scatter diagram of hydrogen adsorption selectivity vs. the differences of isosteric heats of adsorption between $\mathrm{CH}_{4}$ and $\mathrm{H}_{2}$ in the COFs at $298 \mathrm{~K}$ and 35 bar. (b) Scatter diagram 
of volumetric energy density of the COFs vs. isosteric heats of adsorption of $\mathrm{CH}_{4}$ in the $\mathrm{COFs}$ at $298 \mathrm{~K}$ and 1 bar. (Solid lines are merely for trend guide lines)

Fig.5. Scatter diagram of the volumetric energy density of adsorbed HEM in the COFs at 298K: (a)1 bar (b) 35 bar (c) 80 bar vs. the volumetric geometric surface area of all the COFs. (Solid lines are merely for trend guide lines)

Fig.6. (a) Scatter diagram of the gravimetric energy density of adsorbed HEM in the COFs at 298K, 80 bar vs. the porosity of the COFs; (b) Scatter diagram of the volumetric energy density of adsorbed HEM in the COFs at $298 \mathrm{~K}, 80$ bar vs. the porosity of the COFs; (c) Scatter diagram of the hydrogen adsorption selectivity in the COFs at $298 \mathrm{~K}, 80$ bar vs. the porosity of the COFs; (d) Scatter diagram of the hydrogen adsorption selectivity in the COFs at 298K, 80 bar vs. the dominant pore size of the COFs. (Solid lines are merely for trend guide lines)

Fig.7. The volumetric energy density isobars of HEM adsorbed in 3 COFs: COF-103, COF-108 and NPN-1 at different pressures: (a) 1 bar, (b) 35 bar, (c) 80 bar, with the temperature changing from $250 \mathrm{~K}$ to $350 \mathrm{~K}$. (d) Hydrogen adsorption selectivity variation with the temperatures changing from $250 \mathrm{~K}$ to $350 \mathrm{~K}$ at 35 bar.

Fig.8. The volumetric energy density of HEM adsorbed in 3 COFs: COF-103, COF-108 and NPN-1 with 9 different initial hydrogen bulk feed fraction (changing from $10 \%$ to $90 \%$ ) at 
different pressures: (a) 1 bar, (b) 35 bar, (c) 80 bar, at 298K. (d) Hydrogen adsorption selectivity and adsorbed hydrogen content (by volumetric energy) with 9 different initial hydrogen bulk feed fraction (changing from 10\% to 90\%) at 35 bar and 298K. (HS stands for hydrogen selectivity, $\mathrm{HC}$ stands for hydrogen content in volumetric energy) 

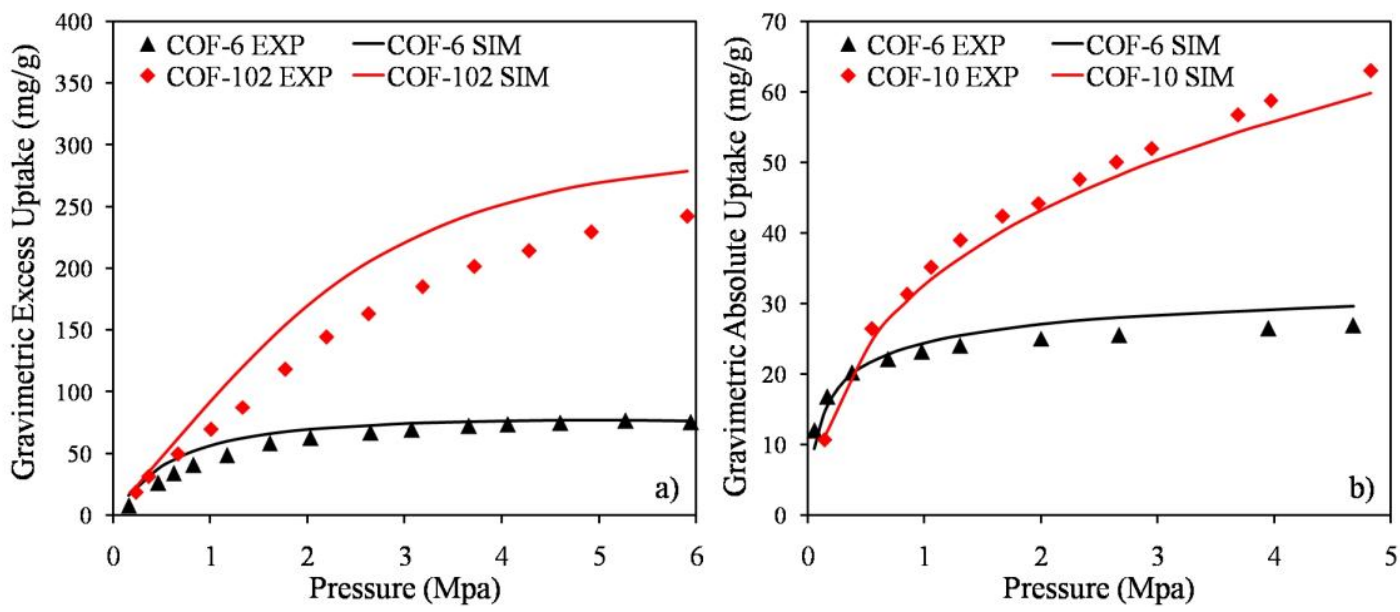

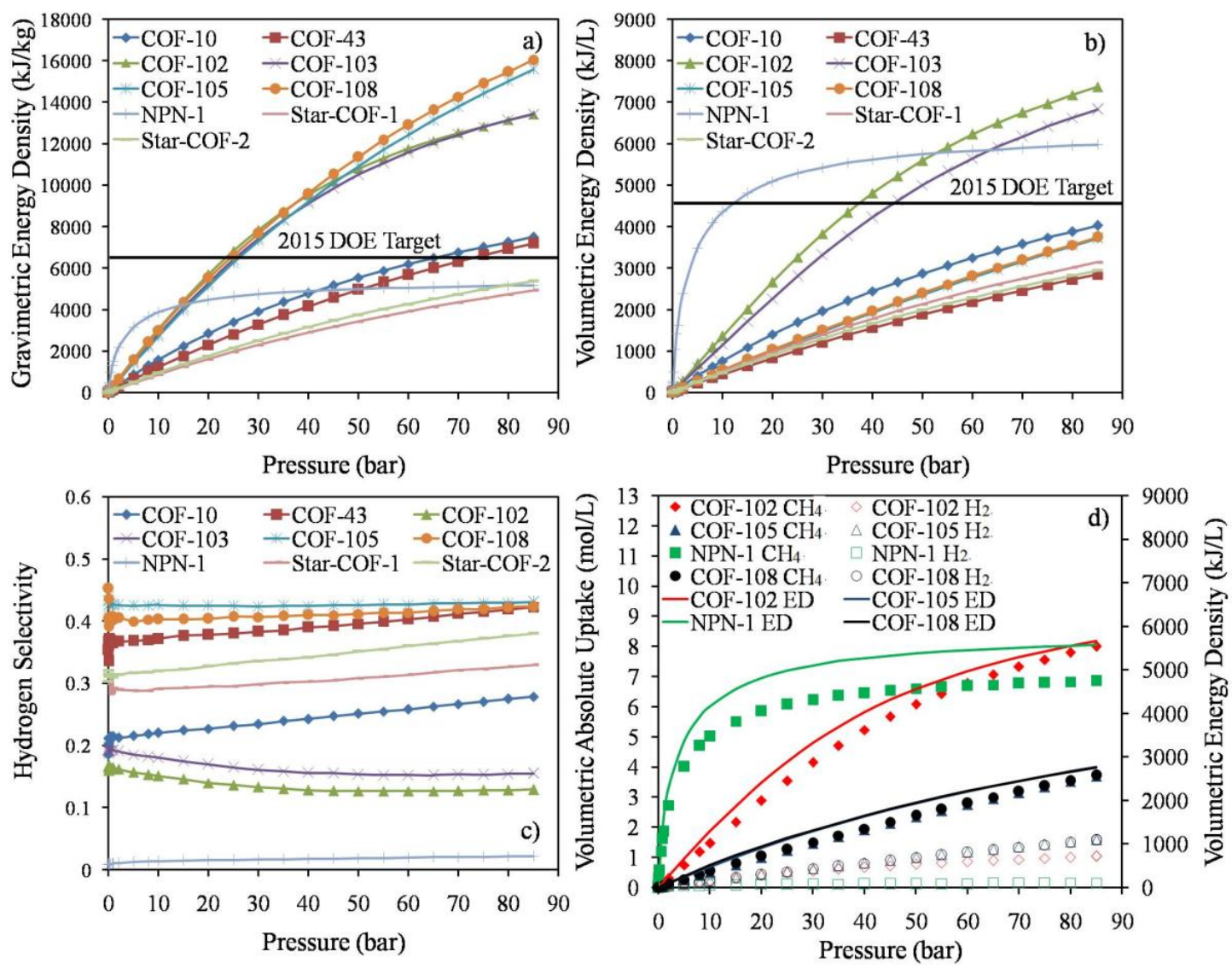

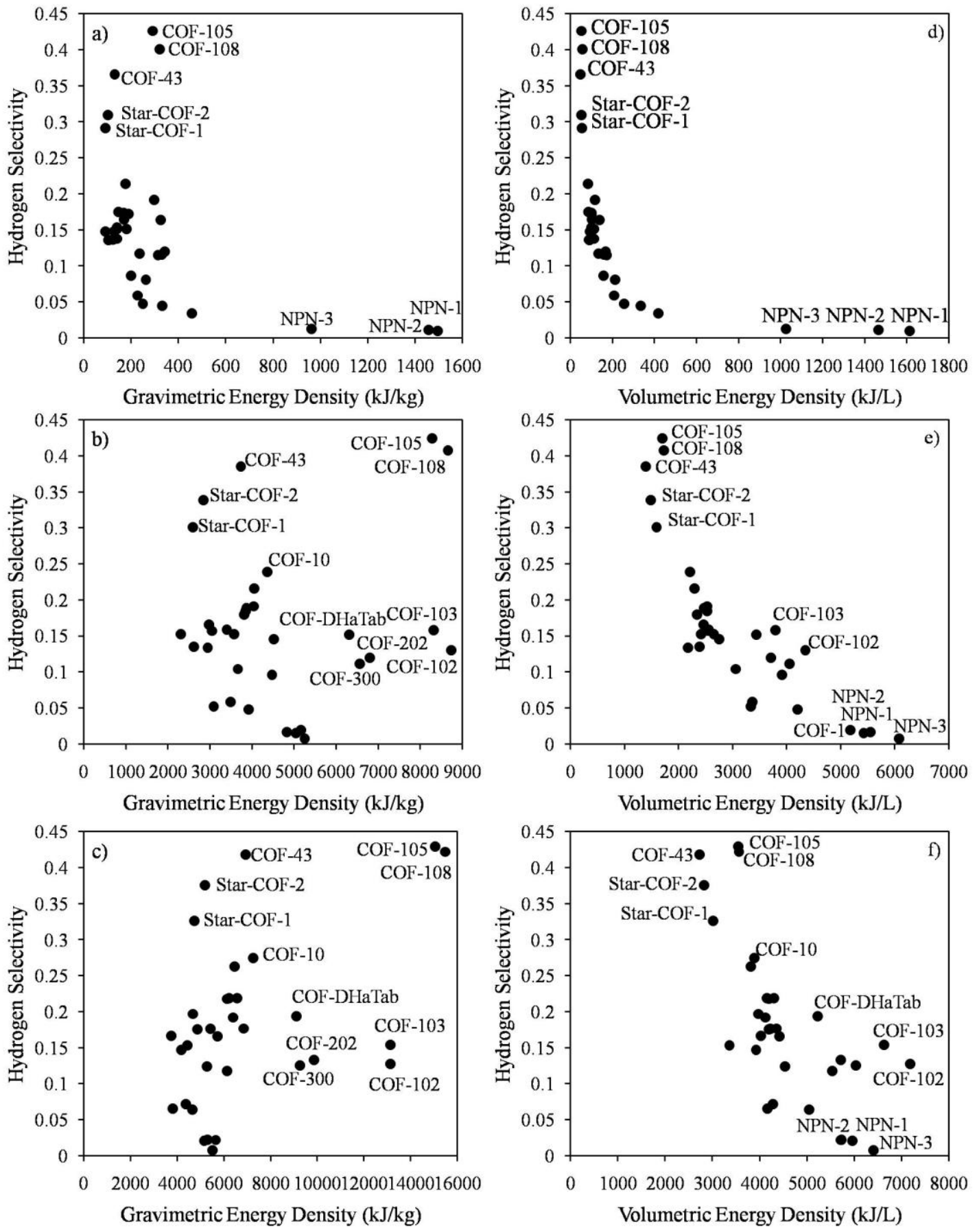

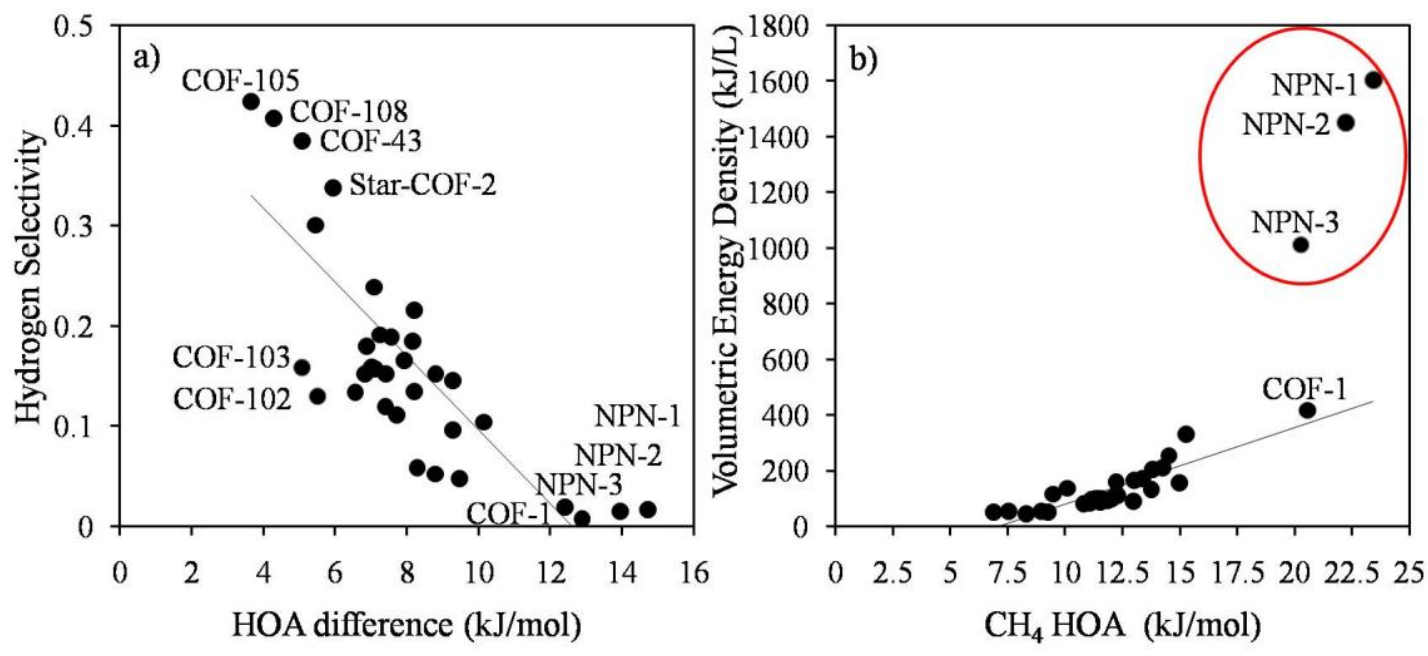

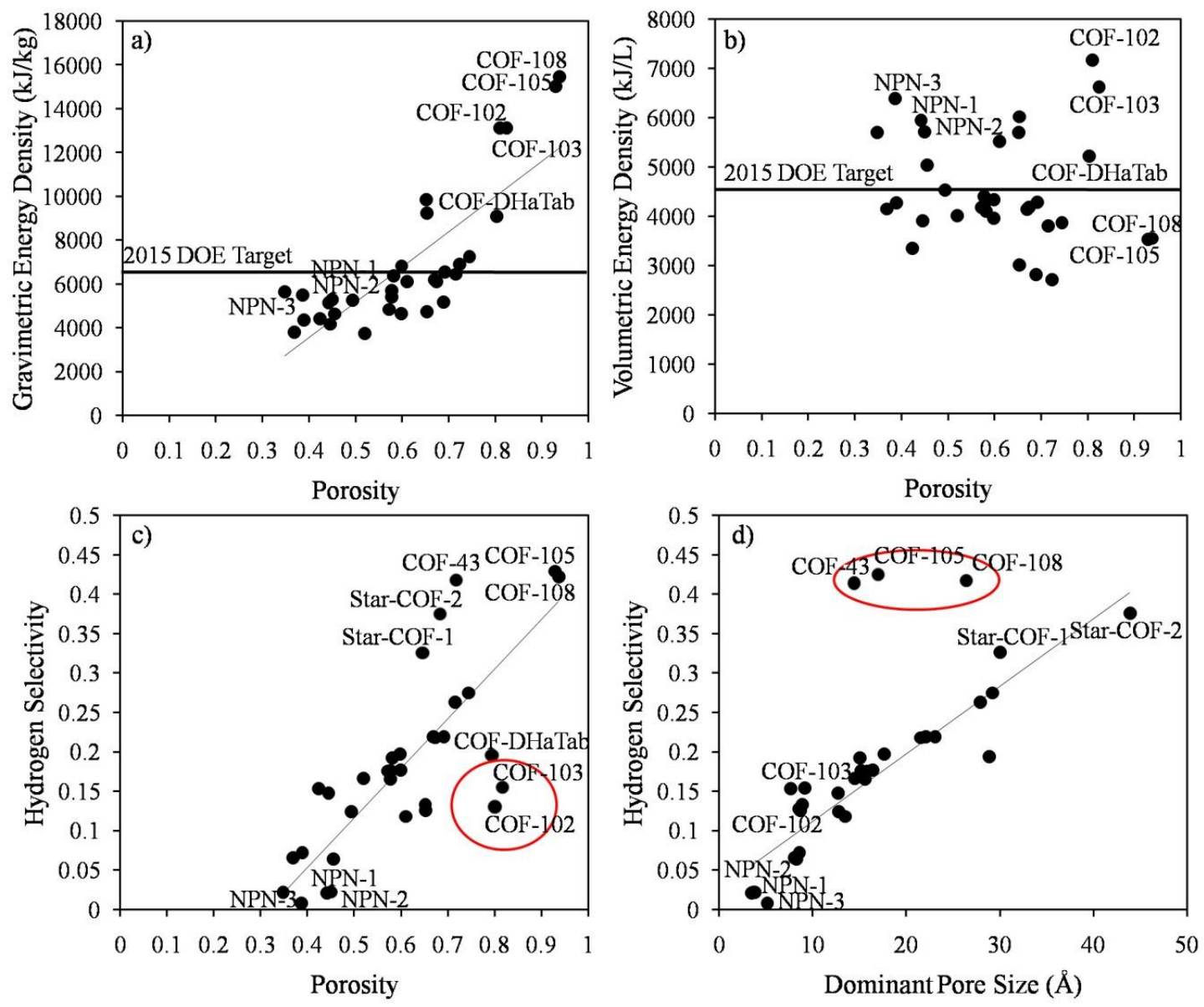

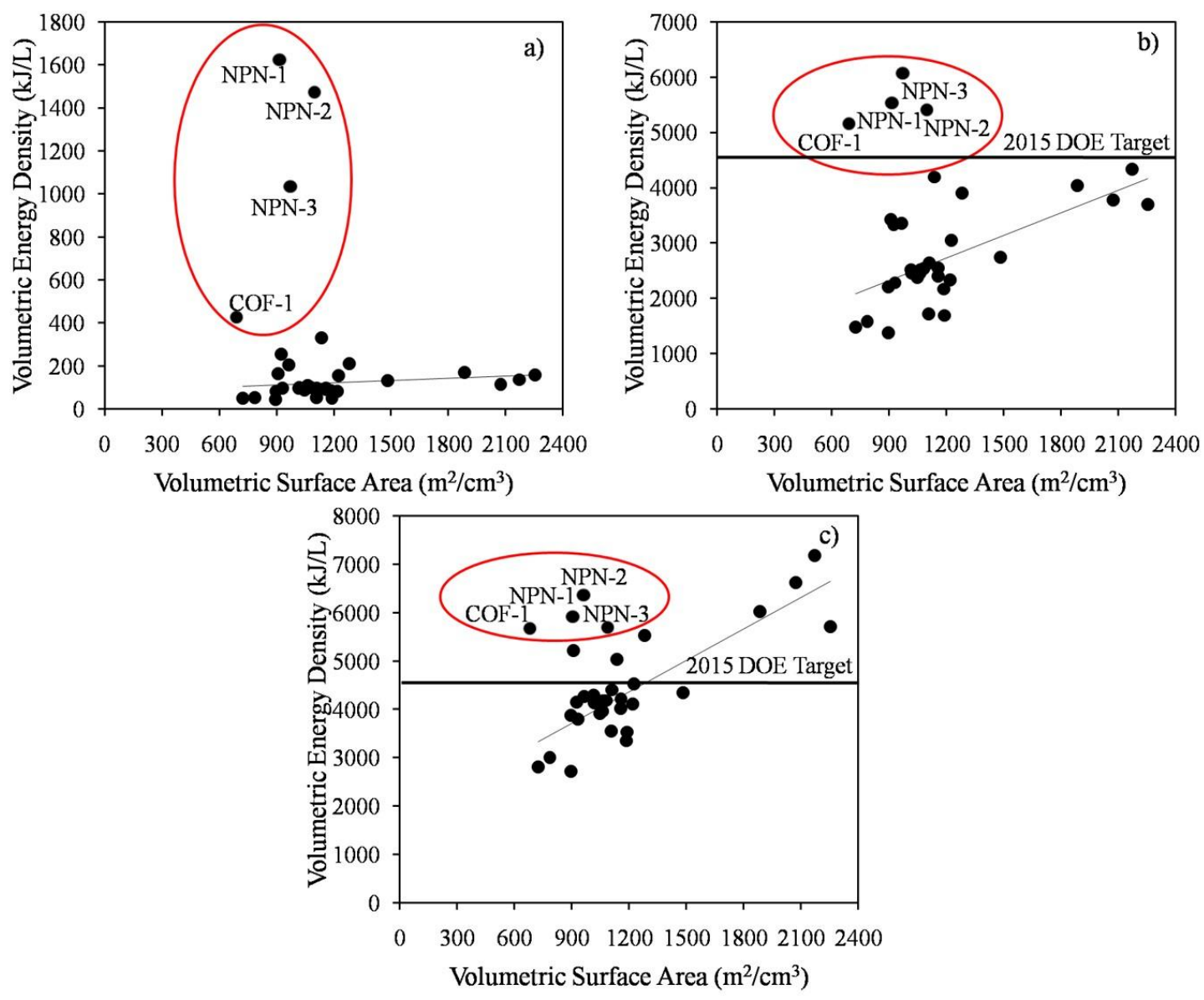

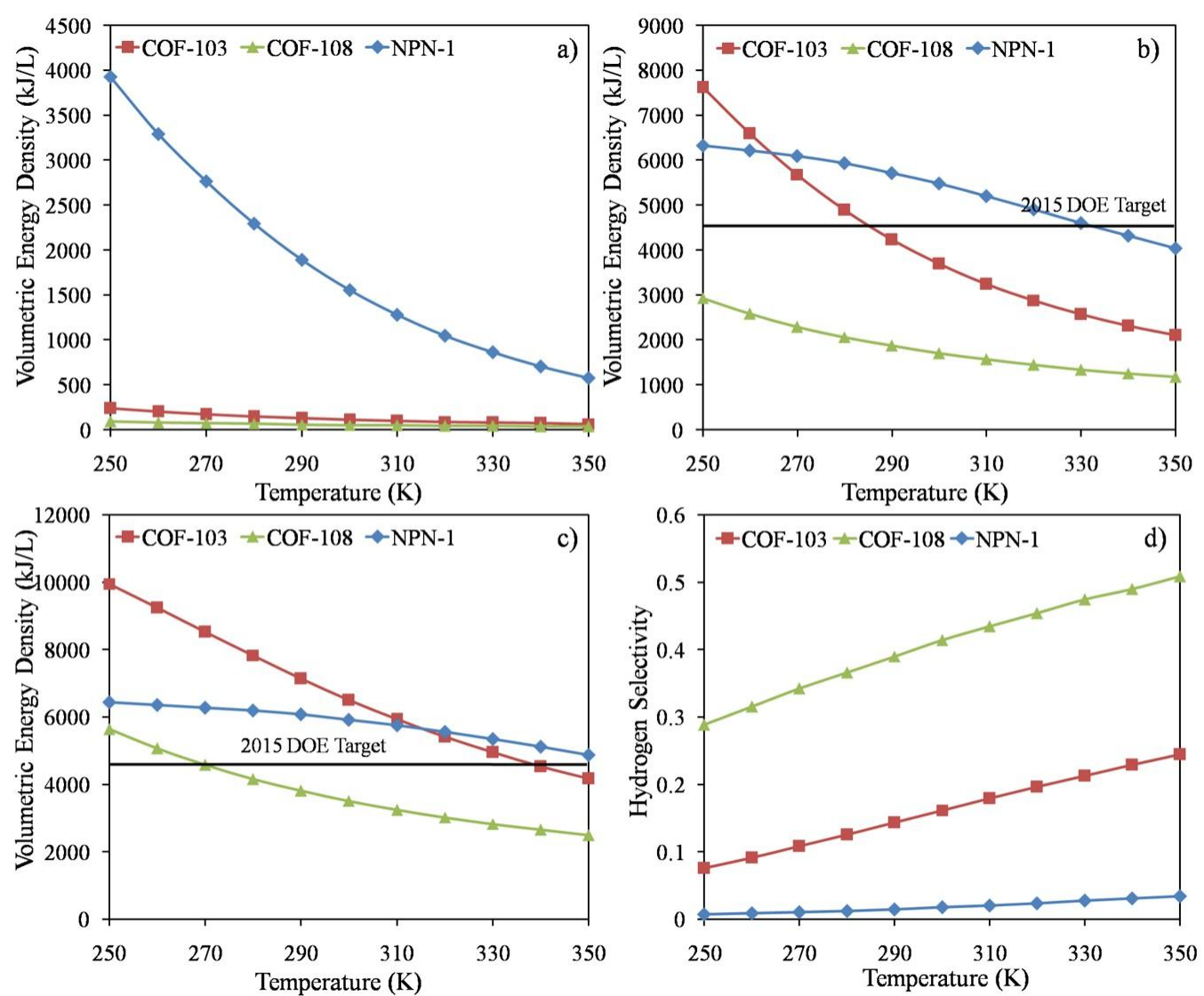


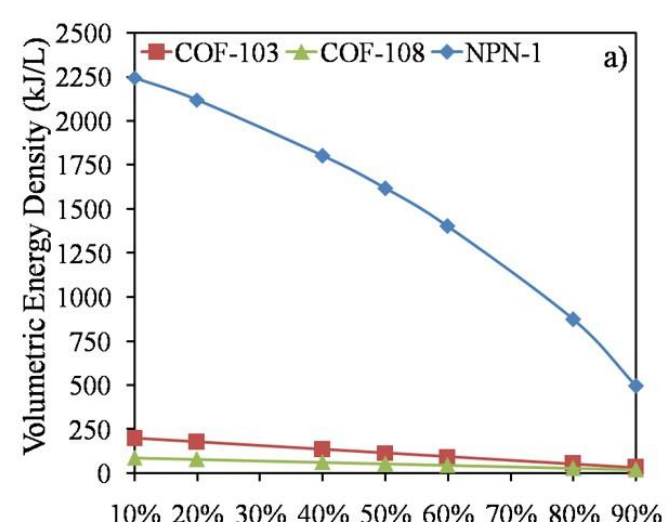

Hydrogen Bulk Composition

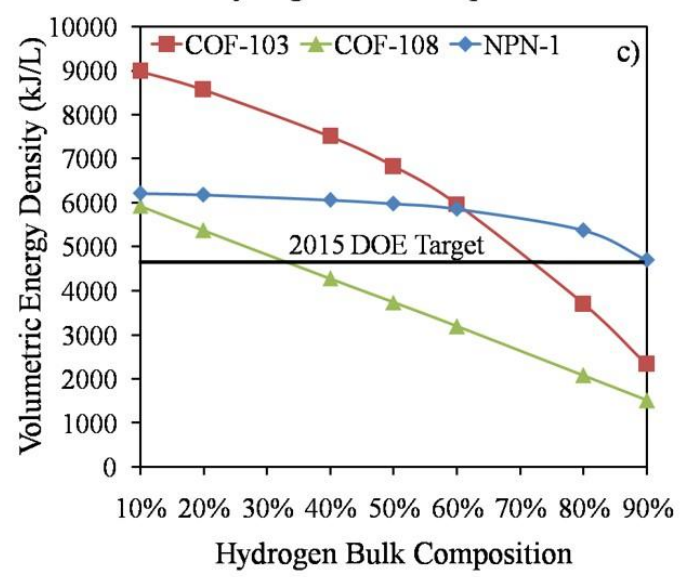

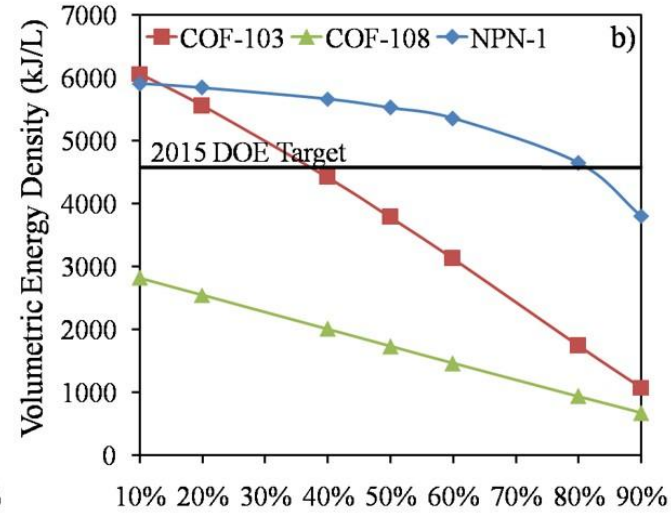

Hydrogen Bulk Composition

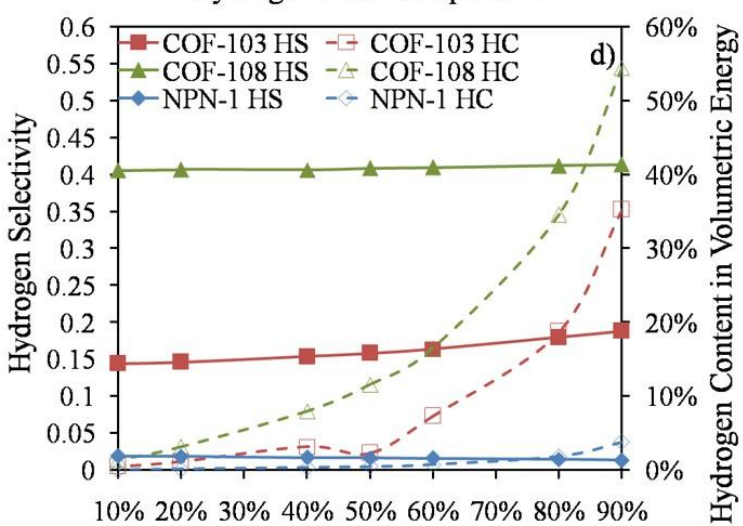

Hydrogen Bulk Composition 

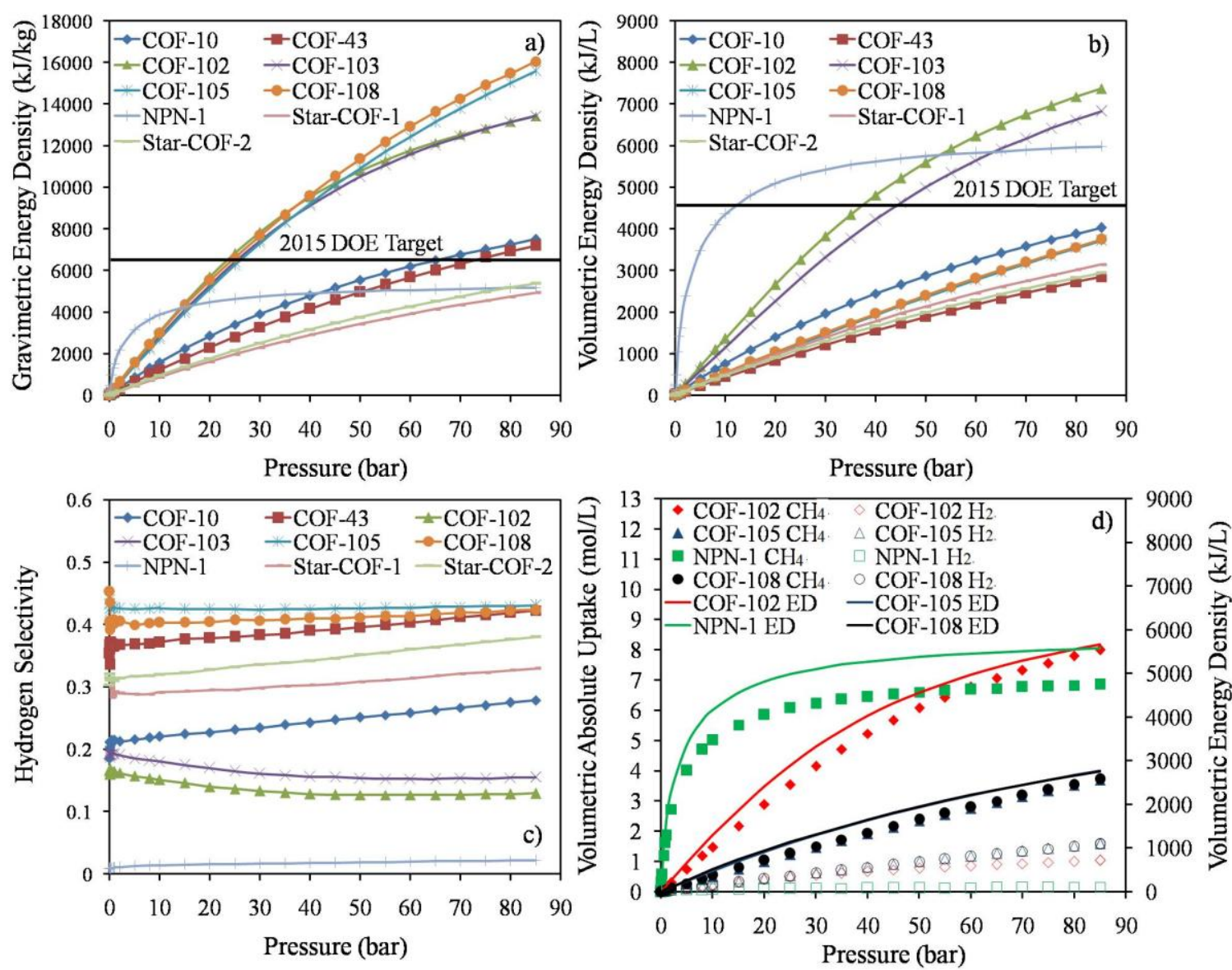